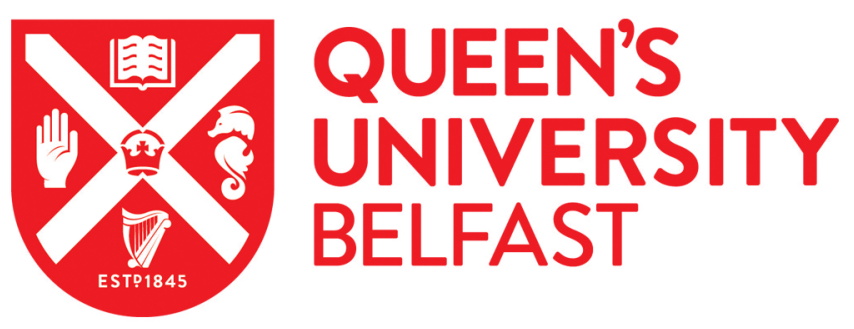

\title{
Cobalamin Concentrations in Fetal Liver Show Gender Differences: A Result from Using a High-Pressure Liquid Chromatography- Inductively Coupled Plasma Mass Spectrometry as an Ultratrace Cobalt Speciation Method
}

Bosle, J., Goetz, S., Raab, A., Krupp, E. M., Scheckel, K. G., Lombi, E., Meharg, A. A., Fowler, P. A., \& Feldmann, J. (2016). Cobalamin Concentrations in Fetal Liver Show Gender Differences: A Result from Using a High-Pressure Liquid Chromatography-Inductively Coupled Plasma Mass Spectrometry as an Ultratrace Cobalt Speciation Method. Analytical Chemistry, 88(24), 12419-12426. https://doi.org/10.1021/acs.analchem.6b03730

Published in:

Analytical Chemistry

Document Version:

Peer reviewed version

Queen's University Belfast - Research Portal:

Link to publication record in Queen's University Belfast Research Portal

Publisher rights

(C) 2016 American Chemical Society.

This work is made available online in accordance with the publisher's policies. Please refer to any applicable terms of use of the publisher.

\section{General rights}

Copyright for the publications made accessible via the Queen's University Belfast Research Portal is retained by the author(s) and / or other copyright owners and it is a condition of accessing these publications that users recognise and abide by the legal requirements associated with these rights.

Take down policy

The Research Portal is Queen's institutional repository that provides access to Queen's research output. Every effort has been made to ensure that content in the Research Portal does not infringe any person's rights, or applicable UK laws. If you discover content in the Research Portal that you believe breaches copyright or violates any law, please contact openaccess@qub.ac.uk. 
1 Cobalamin concentrations in fetal liver show gender an ultra-trace cobalt speciation method

$13{ }^{3}$ Future Industries Institute, University of South Australia Building X, Mawson Lakes

14 Campus Mawson Lakes, SA-5095, Australia

$15{ }^{4}$ Institute for Global Food Security, Queen’s University Belfast, Malone Rd, Belfast

16 BT9 5BN, UK

17 Institute of Medical Sciences, School of Medicine, Medical Sciences \& Nutrition,

18 University of Aberdeen, Aberdeen AB25 2ZD, UK

19

20

21 


\section{ABSTRACT}

23

24

25

26

27

28

29

30

31

32

33

34

35

36

37

38

39

40

41

42

43

Maternal diet and lifestyle choices may affect placental transfer of cobalamin (Cbl) to the fetus. Fetal liver concentration of $\mathrm{Cbl}$ reflects nutritional status with regards to vitamin B12, at low concentration current Cbl measurement methods lack robustness. An analytical method based on enzymatic extraction with subsequent RP-HPLC separation and parallel ICP-MS and ESI-Orbitrap-MS to determine specifically Cbl species in liver samples of only 10-50 mg was developed using 14 pig livers.

Subsequently 55 human fetal livers were analyzed. HPLC-ICP-MS analysis for cobalt (Co) and Cbl gave detection limits of $0.18 \mathrm{ng} / \mathrm{g}$ and $0.88 \mathrm{ng} / \mathrm{g}$ d.m. in liver samples respectively with a recovery of $>95 \%$. Total $\mathrm{Co}\left(\mathrm{Co}_{\mathrm{t}}\right)$ concentration did not reflect the amount of Cbl or vitamin B12 in the liver. Co as Cbl contributes only $45+/-15 \%$ to Cot. XRF mapping and $\mu$ XANES analysis confirmed the occurrence on non-Cbl cobalt in the pig liver hot spots indicating particular Co. No correlations of total cobalt nor Cbl with fetal weight or weeks of gestation were found for the human fetal livers. Although no gender difference could be identified for total Co concentration, female livers were significantly higher in Cbl concentration $(24.1+/-7.8 \mathrm{ng} / \mathrm{g})$ than those from male fetuses $(19.8+/-7.1 \mathrm{ng} / \mathrm{g})(\mathrm{p}=0.04)$. This HPLC-ICP-MS method was able to quantify total $\mathrm{Co}_{\mathrm{t}}$ and $\mathrm{Cbl}$ in fetus liver and it was sensitive and precise enough to identify this gender difference. 
44

45

46

47

48

49

50

51

52

53

54

55

56

57

58

59

60

61

62

63

64

65

66

68

69

\section{INTRODUCTION}

Vitamin B12 or cobalamin (Cbl) is an essential vitamin stored in the liver. ${ }^{1}$ Cbl can occur in different molecular forms from which only two, methyl-cobalamin (Me-Cbl) and adenosylcobalamin (Ado-Cbl), are physiologically active. Me-Cbl is a cofactor for enzymes in the Carbon-1 metabolism, while Ado-Cbl is a cofactor for enzymes involved in 1,2 H-shifts and transfer of an electronegative group to the neighboring carbon atom. Although the majority of vitamin B12 is stored in the liver, the concentrations are at the ultra-trace level. ${ }^{2}$ The methods routinely used to determine vitamin B12 status are either based on microbiological or immunoenzymatic determination of $\mathrm{Cbl}$ in serum and have been criticized for their overestimation or failure to determine low levels of $\mathrm{Cbl}$ and their lack of precision. ${ }^{3,4}$ Analytical methods for Cbl determination based on chromatography coupled to ICP-MS or ESIMS have been reported but so far mainly used for food-supplements. ${ }^{5,6,7,8}$ An analytical method based on thermal acidic denaturation with liquid/liquid extraction of beef liver with subsequent RP-HPLC separation of the main four Cbl species with ESI-MS detection showed promising results for the determination of Cbl in liver samples. ${ }^{9}$ The sensitivity and specificity of this method though needs improving due to the small size of tissue samples available from the human fetus and the extraction needs to be confirmed by using a complementary direct speciation method such as EXAFS and XANES. ${ }^{10}$

The wider aim of this study was to develop a method for Cbl quantitation which is robust but sensitive enough to detect low background levels in human fetal liver samples to determine maternal life-style influence on the fetal nutritional vitamin B12 status. This method was then applied to human fetal liver samples to identify whether vitamin B12 status varies with body weight, gestation age or gender.

This was achieved by the following objectives:

- To test whether it was possible to determine quantitatively the physiologically active forms of $\mathrm{Cbl}$ (Me-Cbl and Ado-Cbl) besides cyano-cobalamin (CN$\mathrm{Cbl}$ ), and hydroxyl-cobalamin (HO-Cbl) individually when spiked to liver in order to evaluate the full conversion of those active forms into $\mathrm{CN}-\mathrm{Cbl}$. 
- To evaluate the quantitative extraction and determination of vitamin B12 and if possible the physiologically active forms of Me-Cbl and Ado-Cbl from pig liver samples using HPLC with parallel detection of using ICP-MS for Co and ESI-MS for the individual Cbl forms.

- To confirm whether extraction was altering the Cbl content by using a direct speciation method for which no extraction is necessary (by XRF (X-ray fluorescence) mapping with subsequent $\mu$ XANES (x-ray absorption near edge spectroscopy).

- $\quad$ To apply the protocol to 55 human fetal livers and quantify the Cbl concentration and $\mathrm{Co}_{\mathrm{t}}$ concentration in fetal livers and evaluate the results with regards to liver weight, sex and gestation age.

\section{EXPERIMENTAL SECTION}

\section{Chemicals and Material}

The different cobalamin (Cbl) standards, Methyl-cobalamin (Me-Cbl), Hydroxycobalamin (HO-Cbl), Adenosyl-cobalamin (Ado-Abl) and cyano-cobalamin (CN-Cbl) (98\%, Sigma-Aldrich Germany) were dissolved in water with a concentration of $1 \mathrm{mg}$ $\mathrm{Co} / \mathrm{mL}$ and stored in the dark. The eluents for the HPLC were $0.1 \%$ formic acid (Analytical reagent grade, Fisher Scientific UK) in water (eluent A) and 0.1\% formic acid in methanol (HPLC grade S, Rathburn UK) (eluent B). Co standards (High purity standards, UK) for calibration were prepared based on a stock solution of $1000 \mathrm{mg} / \mathrm{L}$ diluted with $1 \% \mathrm{HNO}_{3}$, conc. (supra pure, BDH UK). A Rh solution (Specpure, Alfa Aesar Germany) served as internal standard. For the sample preparation different organic solvents were used, including methanol (Laboratory reagent grade, Fisher Scientific UK), acetone (Laboratory reagent grade, Fisher Scientific UK), and additionally ultra-pure water $(18 \mathrm{M} \Omega \mathrm{cm})$. For the liver extraction acetate buffer $(\mathrm{pH}$ 5) (acetic acid: extra pure, Sigma-Aldrich Germany), Papain (from Carica Papaya, Sigma Aldrich Germany), potassium cyanide (Fisher Scientific UK) and $\mathrm{HCl}$ (Laboratory reagent grade, Fisher Scientific UK) were used. Nitric acid conc and hydrogen peroxide (Laboratory reagent grade, Fisher Scientific UK) were used for the 

microwave-assisted digestion of liver samples prior to total Co measurements by ICPMS.

\section{Pig Liver samples}

For the method development 14 pig liver samples were used as a proxy for the human liver samples. The pig livers were bought at a local butcher in Aberdeen and stored at $-20^{\circ} \mathrm{C}$ before analysis.

\section{Human fetal liver}

The collection of fetal material was approved by the NHS Grampian Research Ethics Committees (REC 04/S0802/21). Women seeking elective, medical terminations of pregnancy were recruited with full written, informed consent by nurses working independently at Aberdeen Pregnancy Counseling Service. Only fetuses from normally-progressing pregnancies (determined by ultrasound scan), from women over 16 years of age with a good grasp of English and between 11-21 weeks of gestation, were collected.

Fetuses were transported to the laboratory within 30 minutes of delivery, weighed, crown-rump length recorded, and sexed. Livers were snap-frozen in liquid nitrogen and stored at $-85^{\circ} \mathrm{C}$. All morphological data were from the same study as published in Drake et al. ${ }^{11}$ and are summarized in Table $\mathbf{1 .}$

Table 1: morphological data for mothers and fetuses (mean \pm SE)

\begin{tabular}{|l|c|c|}
\hline & Females & Males \\
\hline Maternal age (yrs) & $25.0 \pm 1.1$ & $23.3 \pm 1.2$ \\
\hline Maternal BMI (m²/kg) & $24.6 \pm 1.1$ & $25.5 \pm 0.9$ \\
\hline $\mathbf{N}$ & 25 & $68.6 \pm 11.1$ \\
\hline Fetal weight (g) & $122.4 \pm 19.3$ & $95.1 \pm 4.5$ \\
\hline $\begin{array}{l}\text { Fetal crown-rump } \\
\text { length (mm) }\end{array}$ & $111.2 \pm 6.3$ & $14.1 \pm 0.3$ \\
\hline $\begin{array}{l}\text { Fetal age (weeks of } \\
\text { gestation) }\end{array}$ & $15.7 \pm 0.6$ & \\
\hline
\end{tabular}




\section{Experiments and methods}

\section{Optimization of extraction method}

141 All solutions were handled under dim light conditions. Cbl was extracted from

142 homogenized freeze dried liver samples using a method modified from Rappazzo et al.

143 (12). To $50 \mathrm{mg}$ liver $10 \mu \mathrm{L}$ potassium cyanide solution (1 \% w/v), $500 \mu \mathrm{L}$ buffer (50

144 mol L-1 sodium acetate $\mathrm{pH}$ 5.0) and varying amounts of papain were added. In the

145 optimized final method $5 \mathrm{mg}$ papain was added. The solutions were incubated at

146 different temperatures and for different time periods and centrifuged after cooling in

147 order to optimize the extraction efficiency with regards to total $\mathrm{Cbl}$. The supernatant

148 was stored at $4^{\circ} \mathrm{C}$ in the dark until analysis.

\section{Total cobalt (Cot) determination}

151 20-50 mg pig or human liver samples were weighed accurately in duplicate into a

152 plastic centrifuge tube. Subsequently $2.0 \mathrm{~mL} \mathrm{HNO}_{3}$ were added and left to stand

153 overnight at $25^{\circ} \mathrm{C}$. Hydrogen peroxide $(0.5 \mathrm{~mL})$ and $0.250 \mathrm{~mL}$ of $20 \mathrm{mg} / \mathrm{kg}$ rhodium

154 as internal standard were added and the samples digested in a Mars 5 microwave oven

155 (Matthews Inc, USA). Blanks as well as the listed CRMs were digested in every

156 round of samples as well. Samples were cooled and diluted with deionised water to a

157 final concentration of $2 \%(\mathrm{v} / \mathrm{v})$ nitric acid. Cobalt was measured by high-resolution

158 ICP-MS (Element 2, Thermo Fisher Scientific) at $m / z 59$ at low resolution $(\mathrm{R}=300)$

159 in addition to $\mathrm{m} / \mathrm{z} 103$ for rhodium as the internal standard.

160

\section{Cobalt speciation using HPLC-ICP-MS/ESI-MS}

162 The separation and determination of the 4 cobalamin species was carried out by a 163 reversed phase HPLC coupled to an ESI-MS and ICP-MS using a methanol gradient 164 programme. The ESI-MS was used in positive FTMS-mode. The instrumental 165 parameters are listed in Table S1. To allow quantification the reversed phase HPLC166 ESI-MS was also linked to an ICP-MS. The HPLC flow was split before the UV167 detector with a ratio of 3:1 (ESI-MS: ICP-MS), the continuous internal standard (Rh) 168 used for ICP-MS was added via a T-piece before the ICP-MS nebulizer to correct for 169 matrix changes. Parameters are listed in Table S1 and further description of the split 170 set up can be found by Bluemlein and co-workers. ${ }^{13}$ 


\section{Synchrotron XRF mapping and $\mu$ XANES speciation of Cobalt}

173 Synchrotron based X-ray fluorescence (XRF) was used for mapping Co distribution in

174 shredded freeze-dried pig liver samples. The samples were prepared as thin pressed

175 pellets. Elemental maps were collected at beamline 20-ID (PNC/XOR) at the

176 Advanced Photon Source (APS), Argonne National Laboratory.

177 The electron storage ring operated at $7 \mathrm{GeV}$. A nitrogen cooled $\mathrm{Si}(111)$ double crystal

178 monochromator, calibrated using a cobalt metal foil, was used to generate the X-ray

179 beam. The fluorescence signal was collected using a 13-element Ge detector

180 (Canberra). Four maps of $(1.5 \times 1.5 \mathrm{~mm})$ were obtained by rastering the sample

181 through the $9700 \mathrm{eV}$ beam of $10 \times 6 \mu \mathrm{m}$ with a step size of $20 \mu \mathrm{m}$ and an integration

182 time of $0.3 \mathrm{~s} / \mathrm{step}$. The elemental mapping of trace levels of Co in a high Fe matrix

183 (hemoglobin) is challenging due to the large overlap between the $\mathrm{Fe} \mathrm{K} \beta$ emission line

$184(7,059 \mathrm{eV})$ and the $\mathrm{Co} \mathrm{K} \alpha$ emission line $(6,915 \mathrm{eV})$. Therefore, a script was developed

185 to subtract the contribution of the Fe $\mathrm{K} \beta$ signal from the sum of the Fe K $\beta$ plus Co K $\alpha$

186 signal based on the known ratio of $\mathrm{Fe} \mathrm{K} \beta$ relative to the collected $\mathrm{Fe} \mathrm{K} \alpha$ signal. Areas

187 on the maps, corrected for Fe interference, showing accumulation of Co were

188 investigated using $\mu$ XANES in order to confirm the identification of Co and assess its

189 speciation. Three scans per point of interest were collected, averaged and normalized

190 using Athena. ${ }^{14}$ The spectra obtained were compared to cobalt standards of vitamin

191 B12 (CN-Cbl), coenzyme B12 (Ado-Cbl), methylcobalamin (Me-Cbl),

192 hydroxycobalamin (HO-Cbl) and also $\mathrm{Co}^{+\mathrm{I}}$ and $\mathrm{Co}^{+\mathrm{II}}$ salts.

193

\section{Quality controls and statistics}

195 Blanks as well as CRMs were measured with every batch of the liver digests for total 196 hepatic Co analysis. Certified standard reference materials (NIST RM8415, NRC 197 TORT-2) were used to check reproducibility and accuracy, with both better than +/$1985 \%$. Spiking experiments into the liver sample of Cbl-species were performed to 199 evaluate the integrity of the Cbl species and the accuracy and precision of the Cbl200 determination.

202 Statistical analyses of data were performed using JMP 9.0.2 software (Thomas 203 Learning, London, UK). For method development ANOVA two way tests were 204 performed. For the human liver samples the normality of data distribution was tested 205 with the Shapiro-Wilk test and non-normally distributed data were log-transformed 
and re-checked for normality prior to analysis by ANOVA and Tukey-Kramer HSD and $t$-tests, where data were not normalized, or the variances remained unequal, nonparametric tests were performed (Wilcoxon Test).

\section{Safety}

211 Work with cyanide poses an extra level of risk, which needs to be assessed before 212 starting to work. Especially cyanide should not be poured in acidic solution below pH 2135 to prevent the generation of volatile $\mathrm{HCN}$.

\section{RESULTS AND DISCUSSION}

\section{Separation and detection}

217 A mixture of the $4 \mathrm{Cbl}$ standards in water was measured with the HPLC-ICP-MS. The

$2184 \mathrm{Cbl}$ species were baseline separated on the C8 column with a methanol gradient. All 219 were well retarded and separated within $14 \mathrm{~min}$ and were detected by their Co signal 220 on $\mathrm{m} / \mathrm{z} 59$ by ICP-MS and simultaneously by their molecular peaks $[\mathrm{M}+1]^{+}$and $221[\mathrm{M}+1]^{2+}$ by ESI-MS (Figure 1a-b). It can be seen that the ICP-MS Co response did 222 not change significantly during the chromatographic run although a gradient 223 programme was used (Figure S1). This behavior is in contrast to what has been 224 observed for arsenic or sulphur, ${ }^{15}$ because Co does not benefit from the carbon 225 enhancement effect since it is already fully ionized in the plasma. The response factor 226 for the Orbitrap varied considerably depending on species as indicated in the different 227 peak heights (Figure 1a). Using the elemental calibration (Figure S1a-b) the amount 228 of cobalt can be calculated for each species using the ICP-MS signal, whereas when 229 solely the ESI-MS is used then for every Cbl an individual calibration curve is 230 required. For quantification an external calibration was used with Co element 231 standards $\left(\mathrm{Co}^{2+}\right)$ from 1 to $100 \mu \mathrm{g} \mathrm{Co} / \mathrm{L}$ using the ICP-MS signal. The calculated 232 detection limit for aqueous solutions is about $0.05 \mu \mathrm{g} \mathrm{Co} / \mathrm{L}$ based on 3 times standard 233 deviation of the background noise. This is more sensitive than the methods listed in a 234 recent review. ${ }^{2}$

\section{Stability of the standards over time}

236 In order to assess the stability of cobalamins (objective 1), a comparison between 237 freshly prepared and stored (frozen) solutions was performed (Figure 1b and 1c). 
238 When the standards were stored for more than a day in a freezer, species transformation took place. CN-Cbl was stable, while Me-Cbl and Ado-Cbl showed only recoveries of $5.2 \%$ and $10.6 \%$ respectively. The overall recovery was however around $90 \%$, since the unstable species transformed to $\mathrm{HO}-\mathrm{Cbl}$, which almost tripled in concentration (280\%). This confirms the recent study of Szterk et al. (9) who found that these transformations may be through oxidation in air and UV radiation, which result in the conversion of all physiologically important species to HO-Cbl. Hence, the samples need to be measured immediately after extraction.

\section{Stability of cobalamin species in different extractant solutions}

247 To extract the different $\mathrm{Cbl}$ species from liver, they have to be liberated from their transport-proteins and transferred, unchanged, into the extract and the majority of the matrix should be removed. Several possibilities were tested for their influence on Cblspeciation by spiking experiments using pig liver in order to address objective 1 and 2 . Treatment of Cbl-standards with papaine, diluted nitric acid, methanol or acetone resulted in species transformation. When all Cbl species were heated in acetone or methanol in order to precipitate all proteins all Cbl species eluted in the void and did not show the corrin-ring moiety (evident through missing $[\mathrm{M}+\mathrm{H}]^{+}$data), this means transformation to unbound polar Co species took place (early eluting Co compounds close to the void volume). Hot water extraction at $50^{\circ} \mathrm{C}$ of the pig liver with subsequent measurement of Co speciation showed that part of Ado-Cbl in the pig liver was stable during this extraction (Figure S2). The majority of Co eluted however in the void (Figure S3). Spiking of all four Cbl species into the pig liver sample revealed also that the Me-Cbl and Ado-Cbl transferred mainly to HO-Cbl rather than unbound not retarded Co. Hence, the reliable quantification of the two bioactive $\mathrm{Cbl}$ species was not possible. Since the aim is to have a sensitive method for total $\mathrm{Cbl}$ in contrast to any non-Cbl (inorganic $\mathrm{Co}$ ), we tested the $\mathrm{CN}-\mathrm{Cbl}$ method when all the Cbl species should be converted quantitatively as CN-Cbl (Figure S4). This method was originally developed for the extraction of Cbl species from serum (2). Quantitative conversion was tested by spiking pig liver with about $4 \mu \mathrm{g}$ Co/g of all four Cbl species in triplicates, and study their stability in the KCN liver extracts. 

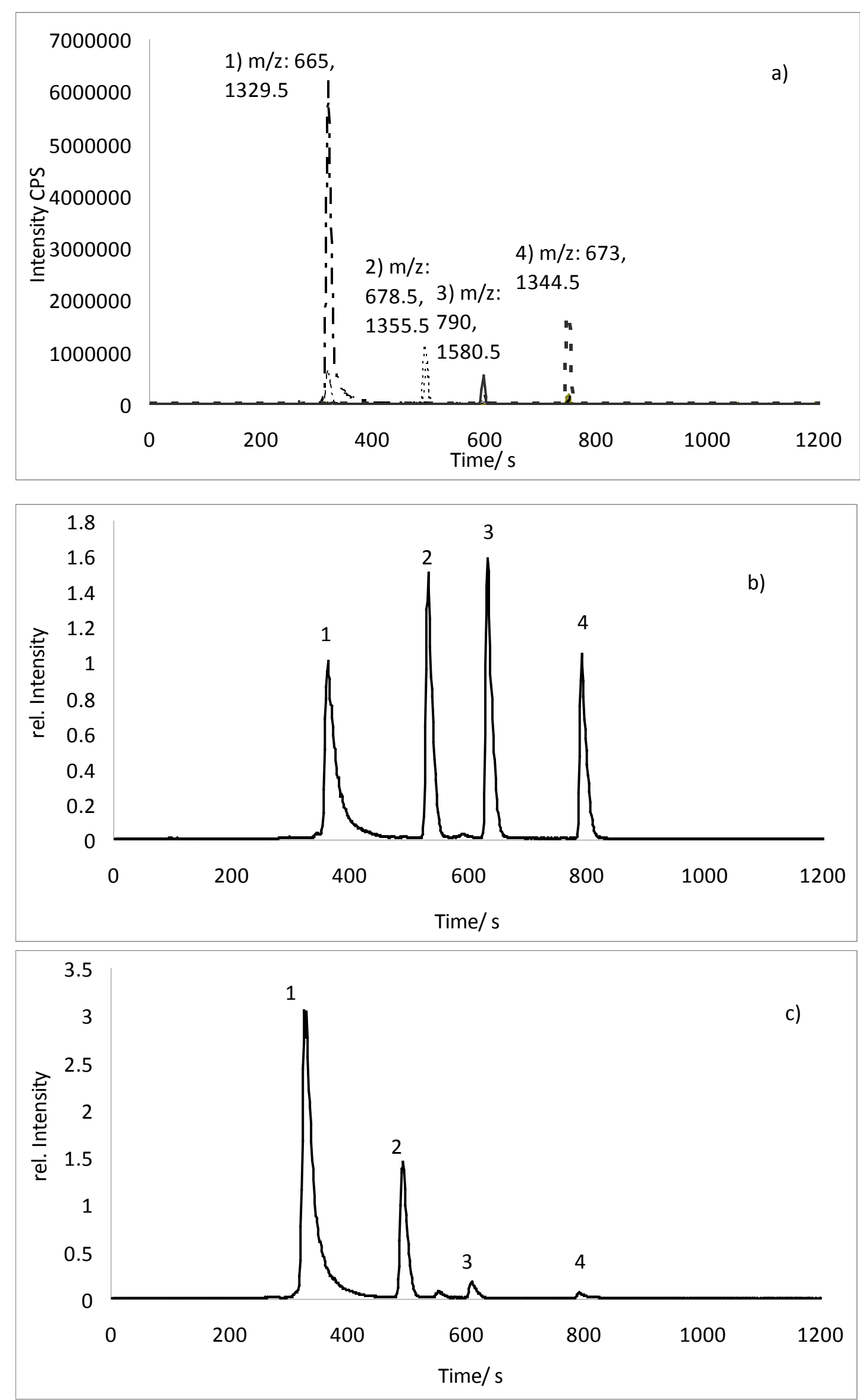
Figure 1a-c: Chromatographic separation of four different cobalamin species using reverse-phase HPLC detected on their $(\mathrm{M}+\mathrm{H})^{+}$and $(\mathrm{M}+2 \mathrm{H})^{2+}$ by ESI-Orbitrap MS (a) and simultaneously on $\mathrm{m} / \mathrm{z} 59$ for cobalt by ICP-MS (b) within 13 minutes. Peaks are 1) HO-Cbl (m/z 665, 1329.5), 2) CN-Cbl (m/z 678.5, 1355.5), 3) Ado-Cbl (m/z 790, 1580.5), 4) Me-Cbl (m/z 673, 1344.5). The degradation of a standard under oxygenated conditions at room temperature is shown in c).

To minimize the risk of underestimation of $\mathrm{Cbl}$ in liver samples Ado-Cbl and Me-Cbl were not determined as their individual species but rather than quantitatively converted to $\mathrm{CN}-\mathrm{Cbl}$ by the addition of sufficient cyanide. Additionally only one peak needs to be integrated which would make the SOP easier and lowers the error. When extracted with the aid of cyanide the resulting chromatogram shows only two Co peak, one for unbound early eluting $\mathrm{Co}$ and one for $\mathrm{CN}-\mathrm{Cbl}$ as illustrated in Figure 2. None of the Cbl species seems to loose Co under the tested conditions. The spiked pig liver did not show an increase in the early eluting (unbound) Co, and only one prominent Co peak, that of CN-Cbl (Figure 2). The column recovery was around $95 \%$. The conversion of all spiked $\mathrm{Cbl}$ species to $\mathrm{CN}-\mathrm{Cbl}$ was quantitative (94 +/- 2\%; $\mathrm{n}=3$ ) which render this method to be accurate. Although the recovery of the spiked Cbl species was quantitative the extraction of $\mathrm{Co}_{t}$ was not (Figure S5). The $\mathrm{Co}_{\mathrm{t}}$ concentration of the unspiked pig liver was $57+/-4.7$ ng Co $\mathrm{g}^{-1} \mathrm{~d}$.m. (Figure S6) while the $\mathrm{Co}_{\mathrm{t}}$ determined in the extract was only $32+/-1.7 \mathrm{ng} \mathrm{Co} \mathrm{g}^{-1} \mathrm{~d}$.m., hence the extraction efficiency of $\mathrm{Co}_{\mathrm{t}}$ was only $56 \%$. Although the nature of the unaccounted Co species was unknown the extraction method for Cbl was further optimized in order to prevent potential loss of Cbl species in the liver samples by varying the papain amount, the temperature and incubation time. The optimized extraction efficiency was $71 \pm 28 \%(\mathrm{n}=4)$ of cobalt using between 10-50 mg liver $5 \mathrm{mg}$ papain with $3 \mathrm{~h}$ incubation at $37^{\circ} \mathrm{C}$. (Figure S5). Although the spiked Cbl gave an excellent precision of $+/-2 \%$, the precision of the intrinsic $\mathrm{Co}_{t}$ in the liver was higher $(+/-40 \%)$ at the level of $4 \mu \mathrm{g} \mathrm{Co} \mathrm{as} \mathrm{Cbl} / \mathrm{g}$. This indicate that the liver samples were not homogeneous with regards to the $\mathrm{Co}_{\mathrm{t}}$ when only $10-50 \mathrm{mg}$ samples were taken. Hence, the homogeneity of the sample was investigated by using the XRF mapping (objective 3).

Using 3 times the standard deviation (SD) of the blank level, and a sample mass of 50 mg d.m., the method for has a detection limit of $0.18 \mathrm{ng} / \mathrm{g} \mathrm{d.m}$. for $\mathrm{Co}_{\mathrm{t}}$, while for the speciation for total Cbl a lower detection limit of $0.88 \mathrm{ng} \mathrm{Co/g} \mathrm{d.m.} \mathrm{was} \mathrm{established.}$ A practical lower limit of quantification (10 times the SD of the blank) is therefore 
about $3 \mathrm{ng}$ Co as Cbl/g d.m. liver. This means that the described analytical method was capable to detect between 10-50 pg Co as Cbl (depending on the weight of the sample). This is superior to all so far described methods. ${ }^{2,9,12}$ This should be lower than the expected levels of those analytes in human fetal liver.

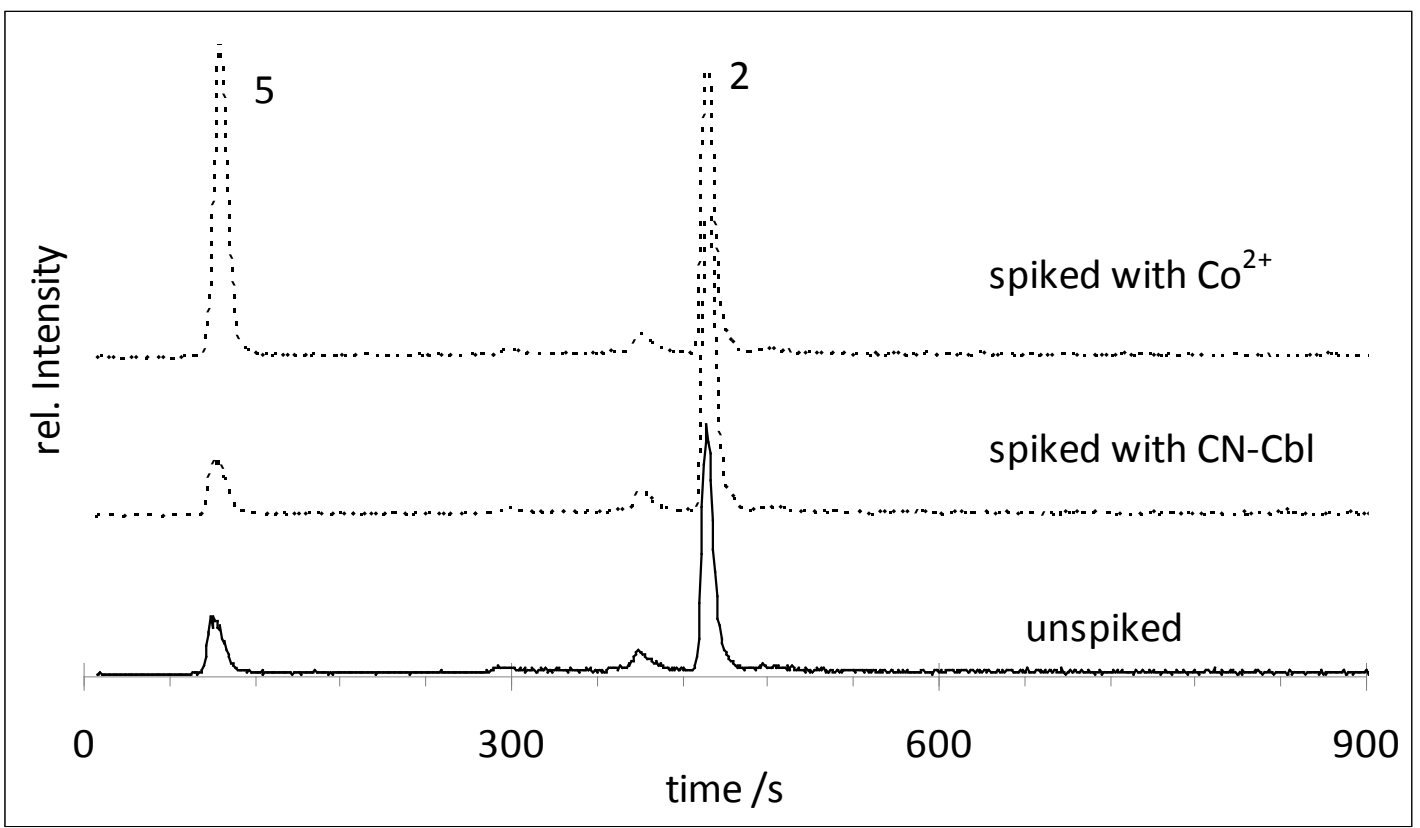

Figure 2: HPLC-ICP-MS chromatogram shows a pig liver extract using cyanide of an unspiked and a $\mathrm{CN}-\mathrm{Cbl}$ (peak 2) and $\mathrm{Co}^{2+}$ as nitrate spiked extracts (peak 5) gives the inorganic cobalt in the extract, while peak 2 shows CN-Cbl.

\section{Cobalt speciation in pig liver}

324 The optimized method was applied to 14 pig liver samples. The amount of sample used during this experiment was kept to below $50 \mathrm{mg}$ per sample in order to evaluate the suitability of the method for human fetal samples. $\mathrm{Co}_{\mathrm{t}}$ varied significantly over almost one order of magnitude (18-145 ng Co/g d.m.). The extraction efficiency was measured for a subset ( 2 samples, $n=3$ ) gave $89 \pm 14 \%$ with an outlier of only $43 \%$

329 (Figure S7). All samples $(n=14)$ were extracted for speciation analysis with the

330 optimized method. The extractable Co concentration ranged from 18 - $50 \mathrm{ng} \mathrm{Co} / \mathrm{g}$ 331 d.m. Although the extraction efficiency was nearly quantitative, only a fraction of total Co was in the form of Cbl measured as CN-Cbl (Figure S8). The Cbl fraction accounted for $45 \pm 15 \%$ of Cot, while non-specified unbound cobalt was nearly $55 \%$ 
with little unaccounted non-extractable Co. While $\mathrm{Co}_{t}$ concentration was highly variable the Cbl-concentration was remarkable constant with $23 \pm 8 \mathrm{ng} \mathrm{Co} / \mathrm{g}$ d.m $(n=14)$. Interesting is the variation between the different liver samples; the liver sample CC-5 contained around $68 \%$ of $\mathrm{Co}$ as $\mathrm{Cbl}$ in the extracted material, while L1F had only $25 \%$. This variability has been seen in beef livers before. ${ }^{1}$ Considering only the extractable $\mathrm{Co}$, $\mathrm{Cbl}$ shows a logarithmic trend when related to extractable Co (Figure 3). That indicates that high total Co concentrations in pig livers might not be the result of vitamin B12 accumulation but rather of Co which is not bound as Cbl. This contradicts that the amount of Vitamin B12 linearly depends on the amount of $\mathrm{Co}_{\mathrm{t}}$ in liver reported elsewhere (1). Hence, the Cbl concentration cannot be estimated 344 from the total Co concentration in pig liver. The amount of $\mathrm{Cbl}$ needs to be measured directly in order to give a reliable account of the vitamin B12 concentration.

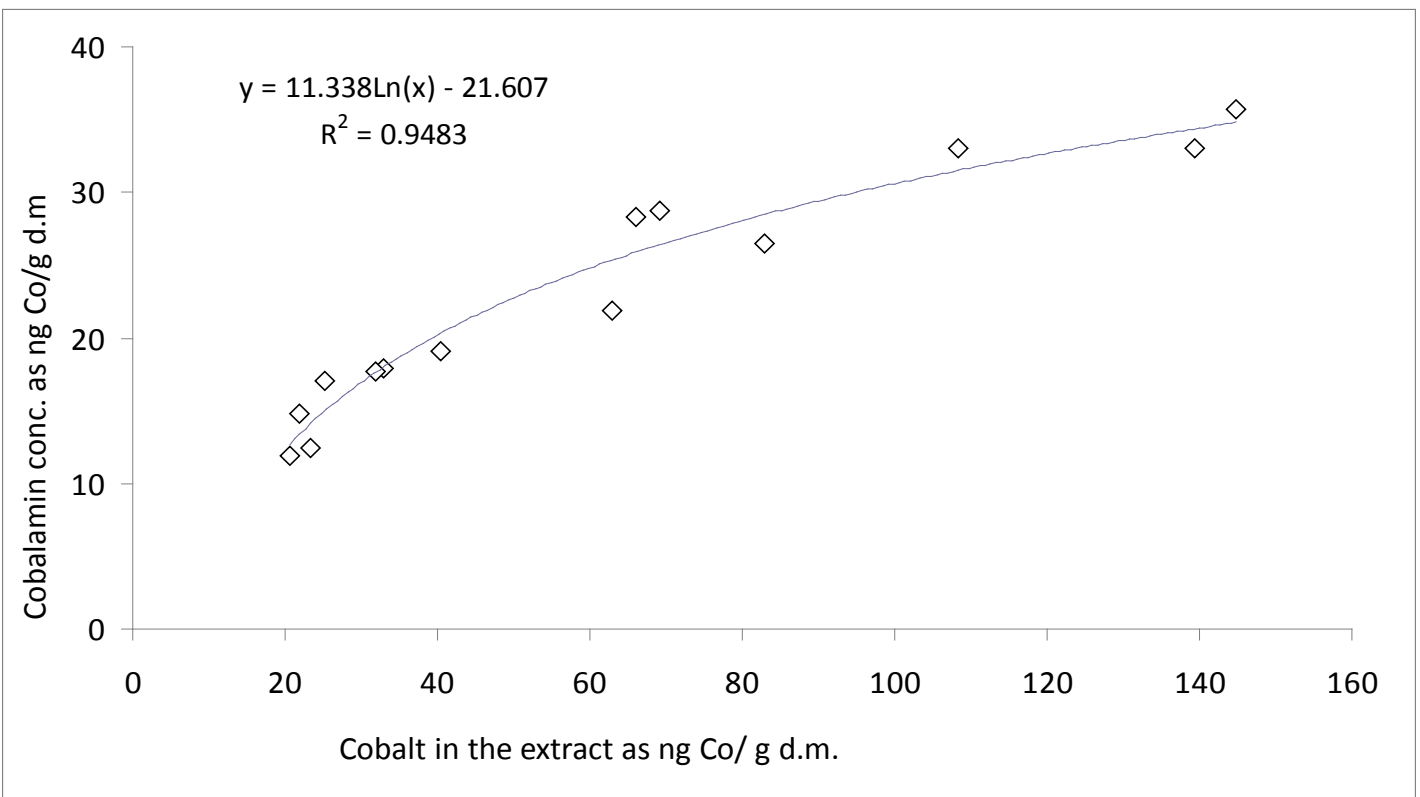

Figure 3: Correlation cobalamin expressed as cobalt versus the cobalt concentration in the extract of 14 pig liver samples.

356 species to CN-Cbl was achieved. The separation of the Co species has been shown to

357 be robust (retention times did not vary more than $0.1 \mathrm{~min}$ ) throughout the analysis.

358 Although pooled samples showed good reproducibility in their Co concentration

359 (approx.. 5\% Figure S5), subsamples taken from individual livers showed 
considerable variability (Figure S6 and S7). This may suggest that cobalt is heterogeneously distributed throughout the pig liver especially if only a small sample is taken, which would be unexpected for physiologically regulated Cbl.

To shed more light on the heterogeneity of $\mathrm{Co}$ and $\mathrm{Cbl}$ in the liver and whether $\mathrm{Cbl}$ species transformation had taken place during the sample preparation, i.e. the release of cobalt from the corrin ring, XRF mapping of the pig liver and subsequent $\mu$ XANES was used for unspiked pig liver samples which showed qualitatively the occurrence of Ado-Cbl. The challenges to overcome were first the low concentration of cobalt $<0.1$ $\mathrm{mg} / \mathrm{kg}$ and the interference of the Fe $\mathrm{K} \beta$ fluorescence, which overlaps with Co K $\alpha$. Therefore Fe and Co were measured simultaneously and every pixel was corrected using Co $\mathrm{K} \alpha-\mathrm{Fe} \mathrm{K} \alpha / \mathrm{K} \beta$ resulting in a cobalt specific map of the liver sample. The results clearly indicated the presence of Co in small hotspots (approximately 10 to 30 $\mu \mathrm{m}$ in size) throughout the samples (Figure 4 and S9). The XANES spectra of the cobalt hotspots seems similar to inorganic $\mathrm{Co}^{+\mathrm{II}}$ and $\mathrm{Co}^{+\mathrm{III}}$ compounds and distinctively different from the XANES spectra of cobalamin standards characterized by a double feature in the main absorption peaks $\left(\mathrm{Co}^{+\mathrm{II} / \mathrm{III}}\right)$. Although the nature of these hotspots are unknown, it is not inconceivable that these hot spots are the result of absorbed cobalt containing particles. This explains would explain the heterogeneity of $\mathrm{Co}_{\mathrm{t}}$ but the homogenous distribution of $\mathrm{Cbl}$. Due to the above mentioned $\mathrm{Fe}$ interferences in these samples, a homogeneously low distribution of cobalamin in the sample would not be detected either by XRF or $\mu$ XANES. However, XANES and XRF analysis suggests that the majority of Co in the pig livers were not in the form of $\mathrm{Cbl}$ but rather in the form on unbound $\mathrm{Co}^{+\mathrm{II}}$. Therefore, this confirmed the relatively low extraction efficiency of $\mathrm{Co}_{\mathrm{t}}$ (70-80\%) combined with the high recovery of spiked Cbl species. Hence, the described methodology with a low limit of detection $(<1 \mathrm{ng}$ Co as Cbl/g d.m.) and its precision of $<5 \%$ and its accuracy of $94 \%$ it was suited to use for the determination of $\mathrm{Cbl}$ in fetal liver samples. 
$\mathrm{CoK} \alpha$

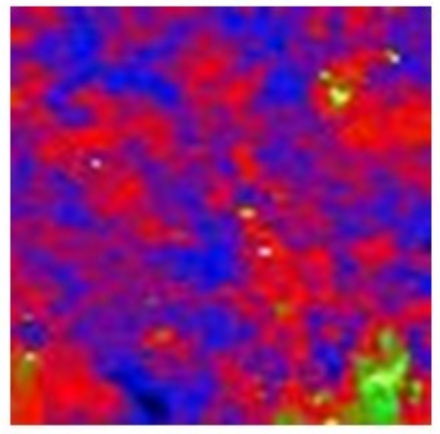

$\mathrm{Fe} \mathrm{K \alpha}$

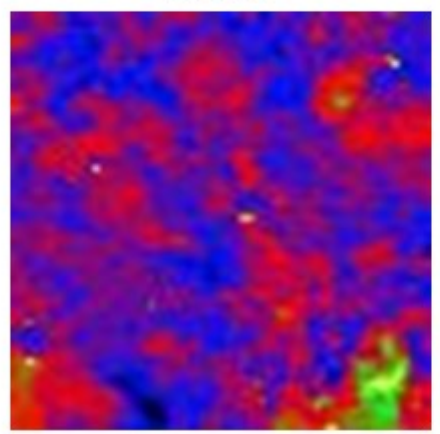

$\mathrm{Co} \mathrm{K \alpha}-\mathrm{Fe} \mathrm{K} \alpha / \mathrm{K} \beta$

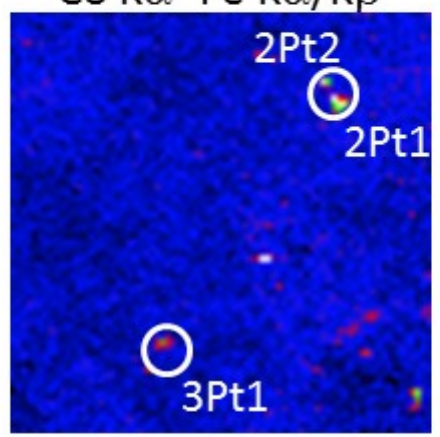

Figure 6

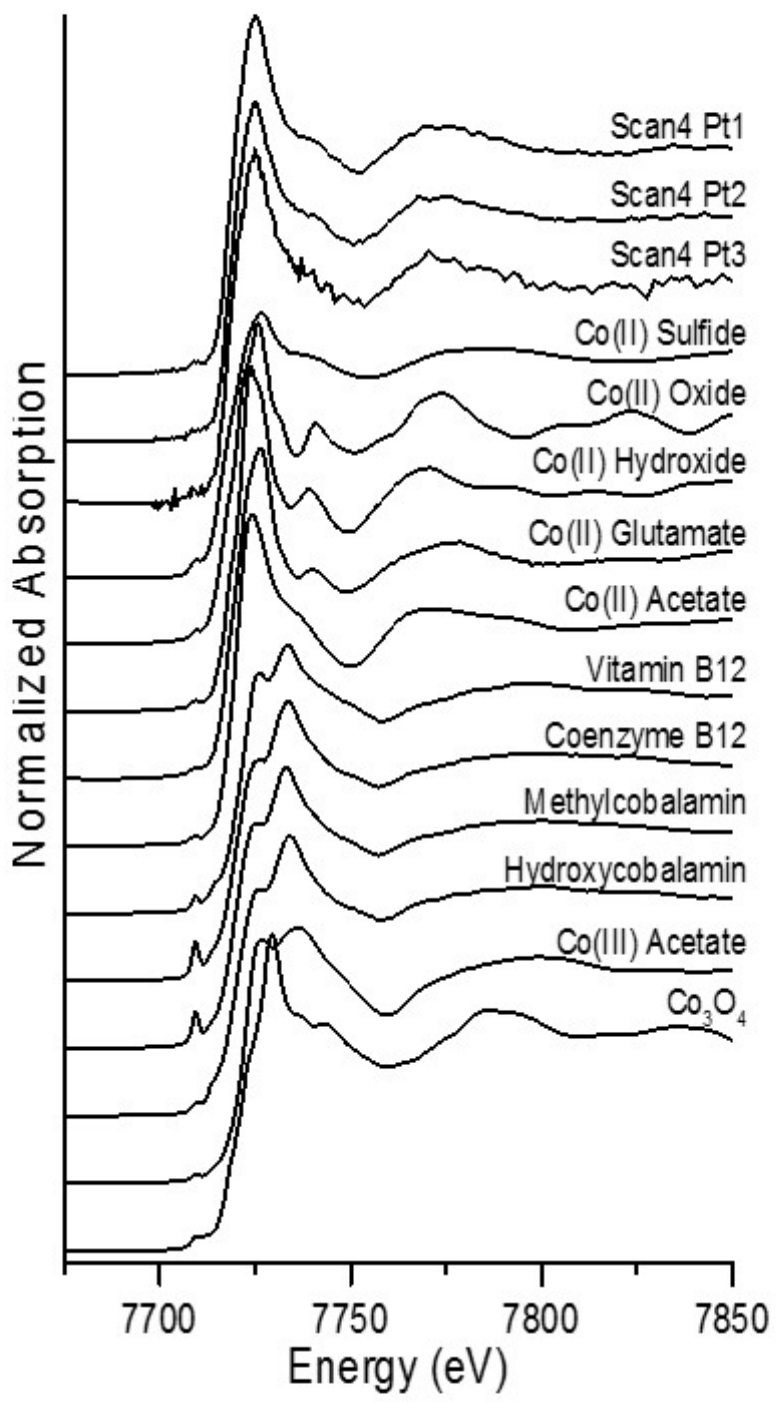

Figure 4a-b: 2 D cobalt map $(1.5$ x $1.5 \mathrm{~mm})(\mathrm{a})$ from a shredded pig liver paste (resolution of about $20 \mu \mathrm{m}$ with the $\mu$ XANES spectra taken at the hotspots (b) in comparison to the XANES spectra of four different cobalamin standards.

\section{Human fetal liver samples}

$\mathrm{Co}_{\mathrm{t}}$ in human fetal samples was analyzed in duplicate and showed a high variability (25 to 190 ng Co/g d.m., detection limit 2 ng/g d.m. Figure 5). All livers had Cot and 
398 Cbl concentrations above the detection limits. The first results of the study has been

399 published partly by Drake et al. ${ }^{11}$ with regards to lifestyle influence on the Cbl

400 concentration in the fetal livers without describing the analytical method in detail.

401 Here, we describe the analytical method capable of measuring Cbl with high

402 sensitivity and precision and subsequent aspects of the study which enabled us to look

403 at a part of the study which was previously not described.

404 There was no correlation between weight and the $\mathrm{Co}_{\mathrm{t}}$ concentration neither was a

405 significant gender difference found (unpaired two-way ANOVA, $\mathrm{p}=0.082$ ). The $\mathrm{Co}_{\mathrm{t}}$

406 concentrations were comparable with those reported in by Caldas and Dorea. ${ }^{1}$ When,

407 however, the Cbl concentration was measured, a significant gender difference could

408 be established (two-way ANOVA, $\mathrm{p}<0.05$ ). The Cbl concentration in the female liver

409 of non-smoking mothers was $643 \pm 48 \mathrm{ng}$ Vitamin B12/g dm, whereas male fetal liver

410 of non-smoking mothers contained $497 \pm 51 \mathrm{ng}$ Vitamin B12/g. The reason why there

411 is a gender difference is still unclear and how the C1 metabolism of the fetus is

412 influenced when the mother smokes has been discussed elsewhere. ${ }^{11}$ The data also

413 indicate that the vitamin B12 concentration correlates linearly with the $\mathrm{Co}_{\mathrm{t}}$

414 concentration in the liver of the fetuses independent on the gender $(\mathrm{P}<0.001)$ (Figure

415 5).However, even if the correlation is significant the variability was still very large

416 within the data set and a precise measurement of Cbl needs to rely on direct

417 measurement rather than interpolation from total Co (Figure 5). 
A Cobalt $\quad$ B Cobalamin $\quad$ C B12
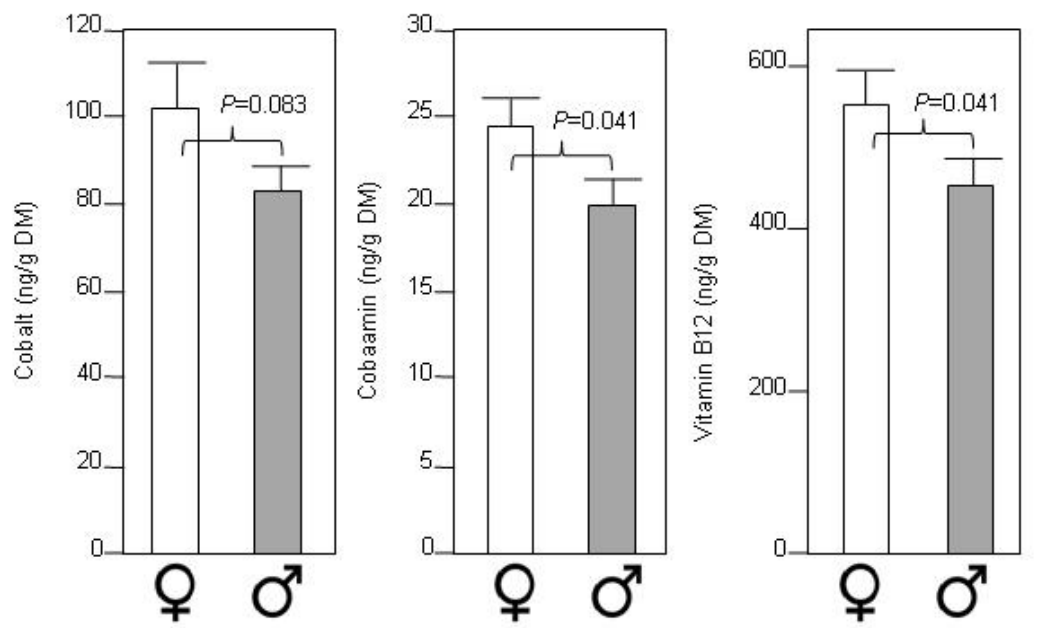

D Cobalt:B12 relationship

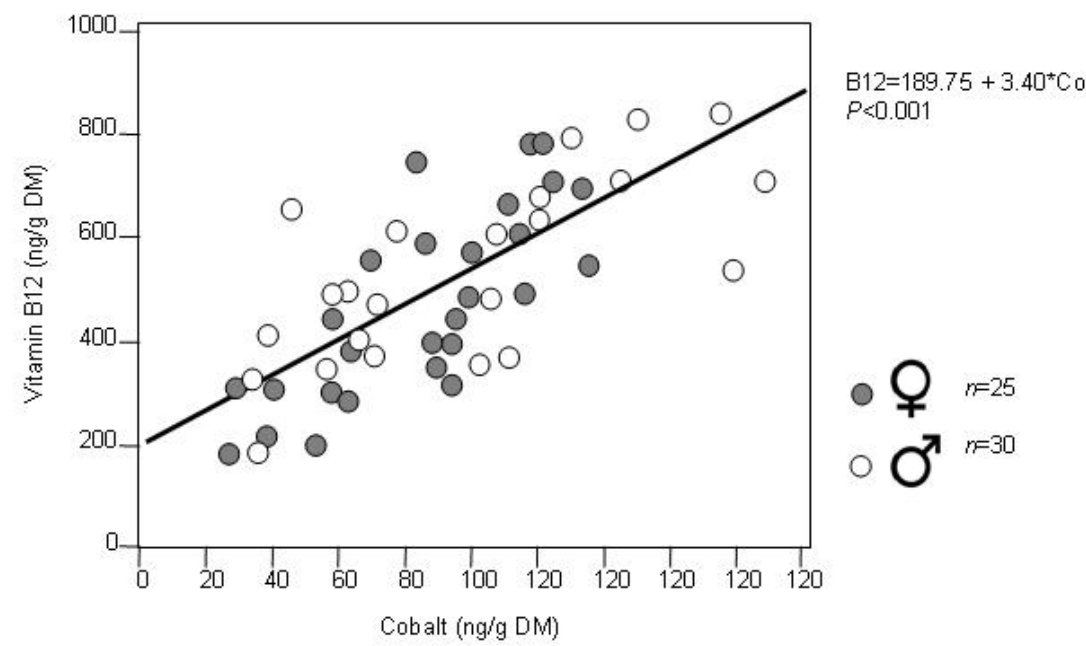

Figure 5: $\mathrm{Co}_{\mathrm{t}}(\mathrm{A})$ and $\mathrm{Cbl}$ concentration (expressed as ng Co/g liver (B) and as ng $\mathrm{Cbl} / \mathrm{g}$ liver $(\mathrm{C})$ in human fetal livers show a significant lower cobalamin level for female fetal livers. The p-values given are based on ANOVA unpaired two way tests. (D) shows the correlation of vitamin B12 and $\mathrm{Co}_{\mathrm{t}}$.

\section{CONCLUSION}

425 The method to determine cobalamin in liver samples described here is sensitive

426 enough to determine background levels of unbound and bound cobalt in fetal liver

427 samples. Although, we were unable to determine the individual physiologically active 
forms of $\mathrm{Cbl}$ (Me-Abl and Ado-Cbl), all forms of Cbl could be transformed into CN$\mathrm{Cbl}$ and determined quantitatively in liver samples with an accuracy of around $94 \%$ and a precision of $+/-5 \%$. A significant amount of Co is in a non-characterized form in the extract, which however is not an artefact of the extraction method and a degradation product of $\mathrm{Cbl}$ species. Not only is $\mathrm{Co}_{\mathrm{t}}$ not representing the amount of Cbl in the liver samples, the analyte is subject to large variability through the accumulation of inorganic Co, which seem to point to particulate Co. The nature of this uncharacterized cobalt needs to be studied in the future.

ACKNOWLEDGEMENTS: S.G and J.B thank the Erasmus exchange program of the EU. Although EPA contributed to this article, the research presented was not performed by or funded by EPA and was not subject to EPA's quality system requirements. Consequently, the views, interpretations, and conclusions expressed in this article are solely those of the authors and do not necessarily reflect or represent EPA's views or policies. Sector 20 facilities at the Advanced Photon Source, and research at these facilities, are supported by the US Department of Energy - Basic Energy Sciences, the Canadian Light Source and its funding partners, and the Advanced Photon Source. Use of the Advanced Photon Source, an Office of Science User Facility operated for the U.S. Department of Energy (DOE) Office of Science by Argonne National Laboratory, was supported by the U.S. DOE under Contract No.

$$
\text { DE-AC02-06CH11357. }
$$

We thank Ms Margaret Fraser and Ms Samantha Flannigan for their expert assistance. The staff at Grampian NHS Pregnancy Counselling Service were essential for collecting fetuses.

Funding: Chief Scientist Office (Scottish Executive, CZG/1/109 (P.A.F.), \& CZG/4/742 (P.A.F.); NHS Grampian Endowments 08/02 (P.A.F.); the European Community's Seventh Framework Programme (FP7/2007-2013) under grant agreement no 212885 (P.A.F.); the Medical Research Council grants MR/L010011/1 (P.A.F.).

\section{References}


${ }^{1}$ Caldas, E.D.; Dorea, J.G. J. Nutri. Biochem. 1992, 3, 539-542.

${ }^{2}$ Kumar, S.S.; Chouhan, R.S.; Thakur, M.S. Anal. Biochem. 2010, 398, 139-149.

${ }^{3}$ Carmel, R. Am. J. Clin. Nutr. 2011, doi: 10.3945/ajcn.111.013441.

${ }^{4}$ Lindenbaum, J.; Rosenberg, I.H.; Wilson, P.W.F.; Stabler, S.P.; Allen, R.H. Am. J. Clin. Nutri. 1994, 60, 2-11.

$\underline{5}$ Chassaigne, H.; Łobiński, R. Anal. Chim. Acta 1998, 227-235.

${ }^{6}$ Kelly, R.J.; Gruner, T.M.; Sykes, A.R. Biomed. Chromatogr. 2005, 19, 329-333.

${ }^{7}$ Jndar Koyyalamudi, R.A.O.; Jeong, S.C.; Yip Cho, K.A.I.; Pang, G. J. Agri. Food Chem. 2009, 57, 6327-6333.

${ }^{8}$ Lipiec, E.; Ruzik, L.; Zhou, Y.; Jarosz, M.; Polec-Pawlak, K. J. Anal. At. Spectrom. 2011, 26, 608-612.

${ }^{9}$ Szterk, A.; Roszko, M.; Malek, K.; Czerwonka, M.; Waszkiewicz-Robak, B. Meat Sci. 2012, 91, 408-413.

${ }^{10}$ Feldmann, J.; Salaun, P.; Lombi, E. Environ. Chem. 2009, 6, 275-279.

${ }^{11}$ Drake, A.J.; O’Shaughnessy, P.J.; Aonteiro, A.; Kerrigan, D.; Goetz, S.; Raab, A.; Rhind, S.M.; Sinclair, K.D.; Meharg, A.A.; Feldmann, J.; Fowler, P.A. BMC

Medicine 2015, 13, Art. Nr. 18

${ }^{12}$ Rappazzo, M.E.; Salmi, H.E.; Hall, C.A. Brit. J. Haematol. 1970, 18, 425-433.

${ }^{13}$ Bluemlein, K.; Raab, A.; Feldmann, J. Anal. Bioanal. Chem. 2009, 393, 357-366.

${ }^{14}$ Ravel, B.; Newville, M. J. Synchrot. Radiat. 2005, 12, 537-541.

${ }^{15}$ Amayo, K.O.; Petursdottir, A.; Newcombe, C.; Gunnlaugsdottir, H.; Raab, A,; Krupp, E.M.; Feldmann, J. Anal. Chem. 2011, 83, 3589-3595. 
Figure 1 